\title{
Recommendations for application of Haemophilus influenzae PCR diagnostics to respiratory specimens for children living in northern Australia: a retrospective re-analysis
}

\author{
Jemima Beissbarth ${ }^{1 *} \mathbb{D}$, Michael J. Binks ${ }^{1}$, Robyn L. Marsh ${ }^{1}$, Anne B. Chang ${ }^{1,2,3}$, Amanda J. Leach ${ }^{1}$ \\ and Heidi C. Smith-Vaughan'
}

\begin{abstract}
Objective: Haemophilus haemolyticus can be misidentified as nontypeable Haemophilus influenzae (NTHi) due to their phenotypic similarities in microbiological culture. This study aimed to determine the prevalence of misidentified NTHi in respiratory specimens from children living in northern Australia.

Results: Among respiratory specimens collected in studies between 2010 and 2013, retrospective PCR analysis found that routine culture misidentified $H$. haemolyticus as NTHi in 0.3\% (3/879) of nasal specimens, 25\% (14/55) of bronchoalveolar lavage and 40\% (12/30) of throat specimens. Therefore, in this population, PCR-based NTHi diagnostics are indicated for throat and bronchoalveolar specimens, but not for nasal specimens.
\end{abstract}

Keywords: Haemophilus haemolyticus (Hh), Nontypeable Haemophilus influenzae (NTHi), Nasopharynx, bronchoalveolar lavage

\section{Introduction}

Nontypeable Haemophilus influenzae (NTHi) disease represents a major global health burden [1]. NTHi are among the most common pathogens identified in the lower airways of patients with chronic lung disease; for example, chronic obstructive pulmonary disease in which up to $90 \%$ of acute exacerbations are associated with NTHi [1]. NTHi are also commonly found in children with otitis media (55-90\%), bacterial conjunctivitis (44-68\%) and $41 \%$ bacterial sinusitis (41\%) [1]. In remote areas of northern Australia, recent cross-sectional data show NTHi in the nasopharynx of $70 \%$ young Indigenous children [2]. In this region, NTHi is the dominant pathogen cultured from the middle ear of children with tympanic membrane perforation [3], and the lungs of children with bronchiectasis [4]. By culture, NTHi

\footnotetext{
*Correspondence: jemima.beissbarth@menzies.edu.au

${ }^{1}$ Menzies School of Health Research, Charles Darwin University, PO Box 41096, Casuarina, NT 0811, Australia

Full list of author information is available at the end of the article
}

from these sites are phenotypically indistinguishable from non-haemolytic strains of the typically commensal $H$. haemolyticus (Hh) [5, 6]. DNA-based methods are required to accurately distinguish NTHi from $\mathrm{Hh}$, and several retrospective studies of presumptive NTHi isolates reported varying rates $(0-27 \%)$ of reclassification of NTHi as Hh [6-8]. Whilst identifying $\mathrm{Hh}$ as a bacteria associated with an infection may not change immediate clinical treatment, understanding the proportion of misidentified NTHi in respiratory samples is important to accurately describe the burden of respiratory disease attributable to NTHi and potentially Hh.

Our aim was to determine the prevalence of misidentified NTHi in 773 specimens from the nasopharynx or nose, ear discharge, throat, and lower airways from 665 children $(<10$ years of age $)$ in northern Australia, and to determine the utility of NTHi PCR diagnostics for our future studies. 


\section{Main text \\ Methods \\ Choice of specimens tested}

Our collection of presumptive NTHi isolates (stored at $-80{ }^{\circ} \mathrm{C}$ ) were originally cultured from nasopharyngeal or nasal (Np), ear discharge (ED), throat, and bronchoalveolar lavage (BAL) specimens collected from children $<10$ years of age living in the north of Australia. All children had participated in previous (2010-2013) cross-sectional studies [2, 4, 9] or a randomised controlled trial [10] (Table 1). The studies providing isolates were purposefully chosen to ensure the isolates from the existing NTHi collection represented a broad age range (0-10 years). Isolates were restricted to collection years 2010-2013 to reduce time dependent bias. In the original studies, culture was performed using standard methods as previously described [3]. Briefly, up to four presumptive NTHi isolates from each specimen were selected on the basis of colony morphology (greyish, transparent, smooth colonies) on chocolate agar and bacitracin-vancomycin-clindamycin-chocolate agar. The isolates were confirmed as presumptive NTHi if hemin and nicotinamide adenine dinucleotide ( $\mathrm{X}$ and $\mathrm{V}$ factor) dependent (Oxoid) and coagglutination negative [Haemophilus Phadebact (10557512, Remel)]. From 773 NTHi-positive specimens (Table 1), 985 isolates were further scrutinised. Of these 773 specimens, 179 (23\%) contributed multiple isolates (between 2 and 4, totalling 391 isolates). Inclusion criteria: specimens collected between 2010 and 2013 in the studies outlined in Table 1 where the participant was of north Australian residence and specimens had at least one presumptive NTHi isolate. No other exclusion criteria were applied. Due to use of a combination of longitudinal and cross-sectional collections, these are only an indication of Hh prevalence.

\section{PCR methods and assays}

Samples were prepared for PCR using a colony lysis method; 1-2 colonies from a subculture of each original NTHi isolate were suspended in $200 \mu \mathrm{l}$ of sterile water, heated at $100{ }^{\circ} \mathrm{C}$ for $10 \mathrm{~min}$, cooled on ice, and centrifuged at 12,000 RPM for 2 min to pellet any cellular debris. We used a Haemophilus protein D (hpd)based PCR assay employing high resolution melt (PCR-HRM; Rotorgene 6000, Corbett Life Science) to discriminate between NTHi and Hh based upon key single nucleotide polymorphisms as described in detail previously [11]. Briefly, the PCR mix comprised $5 \mu \mathrm{l}$ of Bioline $2 \times$ SensiMix SYBR Green (QT650-02), $100 \mathrm{nM}$ each of forward and reverse primer, and $1 \mu \mathrm{l}$ of sample supernatant to a total of $10 \mu \mathrm{l}$ per reaction. Analysis of the HRM was performed on the Rotorgene software (version 1.7). ATCC strains 19418 and 49274 were used as NTHi controls and ATCC 33390 as the Hh control. Samples were genotyped (NTHi or $\mathrm{Hh}$ ) according to their melt curve profile relative to the NTHi or Hh controls. All isolates were run in duplicate, and repeated if amplification curves were not within 0.5 cycles of each other. Isolates that did not amplify by hpd based PCR

Table 1 Source of specimens

\begin{tabular}{|c|c|c|c|c|c|c|c|c|c|}
\hline Study & Age & Study type & $\begin{array}{l}\text { Collection } \\
\text { years }\end{array}$ & Location & $\begin{array}{l}\text { Aboriginal } \\
\text { or Non- } \\
\text { aboriginal }\end{array}$ & $\begin{array}{l}\text { Children } \\
\text { in original } \\
\text { study } \\
\text { (number } \\
\text { NTHi + ve) }\end{array}$ & $\begin{array}{l}\text { Number } \\
\text { of children } \\
\text { NTHi +ve } \\
\text { children } \\
\text { included } \\
\text { in analysis }\end{array}$ & $\begin{array}{l}\text { Specimen } \\
\text { type }\end{array}$ & $\begin{array}{l}\text { Number } \\
\text { phenotypic } \\
\text { NTHi + ve } \\
\text { specimens }\end{array}$ \\
\hline$A^{a}$ & $0-6$ years & Cross-sectional & 2012 & Urban & Both & $\begin{array}{l}564 \\
(185)\end{array}$ & 173 & $\begin{array}{l}\mathrm{Np} \\
\mathrm{ED}\end{array}$ & $\begin{array}{r}172 \\
1\end{array}$ \\
\hline B [2] & $0-3$ years & Cross-sectional & 2010-2012 & Remote & Aboriginal & $\begin{array}{l}444 \\
(328)\end{array}$ & 313 & $\begin{array}{l}\mathrm{Np} \\
\mathrm{ED}\end{array}$ & $\begin{array}{r}308 \\
5\end{array}$ \\
\hline$C[2]$ & $5-8$ years & Cross-sectional & 2010-2012 & Remote & Aboriginal & $\begin{array}{l}138 \\
(133)\end{array}$ & 80 & $\begin{array}{l}N \\
E D\end{array}$ & $\begin{array}{r}78 \\
4\end{array}$ \\
\hline D [10] & 0-7 months & RCT cohort & 2011-2013 & Remote & Aboriginal & $\begin{array}{l}63 \\
(57)\end{array}$ & 57 & $\begin{array}{l}\mathrm{Np} \\
\mathrm{ED}\end{array}$ & $\begin{array}{r}132 \\
5\end{array}$ \\
\hline E [4] & $0-10$ years & Cross-sectional & 2011-2013 & $\begin{array}{l}\text { Urban and } \\
\text { remote }\end{array}$ & Both & $\begin{array}{l}69 \\
(42)\end{array}$ & Total $=665$ & $\begin{array}{l}\text { Np } \\
\text { BAL } \\
\text { Throat }\end{array}$ & $\begin{array}{r}22 \\
30 \\
16 \\
\text { Total }=773\end{array}$ \\
\hline
\end{tabular}


were subsequently confirmed as NTHi using a fucPbased PCR [12]. PCR assays were performed by laboratory staff who were blinded to the identity, age and location of the participants.

We assessed the percent of phenotypic NTHi isolates genotyped as NTHi, Hh or equivocal (unable to be genotyped), the percent of specimens with had concurrent carriage of both and the percent of specimens requiring reclassification (Table 2). Specimens were reclassified as $\mathrm{Hh}$ if all isolates tested (up to four per specimen) were shown to be Hh by PCR-HRM. Data from the five studies were analysed collectively with stratification by the anatomical site of specimen collection. Reclassification isolate and specimen proportions were compared across anatomical sample sites, age group and dwelling (remote versus urban) using the Chi square test (STATA14; StataCorp, USA).

\section{Results and discussion}

Three phenotypic NTHi Np isolates $(0.34 \%, 3 / 879)$ and none of the ED isolates $(0 \%, 0 / 21)$ were reclassified as $\mathrm{Hh}$ by PCR-HRM (Table 2). The three reclassified Hh isolates were from three separate Np specimens, one from each of studies A, C and E. There were too few reclassifications for a valid statistical comparison by the subgroups dwelling and age (Table 3 ). These very low Hh proportions among Np NTHi isolates are in contrast to an American study [6] of children in day care in which $27.3 \%$ of $44 \mathrm{~Np}$ NTHi isolates were reclassified as Hh. In a Western Australian (WA) study of 122 children with recurrent

Table 2 Proportion of NTHi reclassified as Hh, according to specimen type

\begin{tabular}{|c|c|c|c|c|c|c|c|c|}
\hline $\begin{array}{l}\text { Specimen } \\
\text { type }\end{array}$ & $\begin{array}{l}\text { No. } \\
\text { phenotypic } \\
\text { NTHI +ve } \\
\text { specimens }\end{array}$ & $\begin{array}{l}\text { No specimens } \\
\text { with multiple } \\
\text { NTHi } \\
\text { scrutinised }\end{array}$ & $\begin{array}{l}\text { No. } \\
\text { of phenotypic } \\
\text { NTHi isolates } \\
\text { tested }\end{array}$ & $\begin{array}{l}\text { No. isolates } \\
\text { NTHi by HRM } \\
(\%)\end{array}$ & $\begin{array}{l}\text { No. isolates } \\
\text { reclassified } \\
\text { Hh by HRM } \\
(\%)\end{array}$ & $\begin{array}{l}\text { No. isolates } \\
\text { equivocal } \\
\text { by HRM (\%) }\end{array}$ & $\begin{array}{l}\text { No. specimens } \\
\text { with concurrent } \\
\text { NTHi and Hh } \\
\text { by HRM }(\%)^{\text {b }}\end{array}$ & $\begin{array}{l}\text { No. specimens } \\
\text { reclassified Hh } \\
\text { by HRM (\%) }\end{array}$ \\
\hline $\mathrm{Np}$ & 712 & 153 & 879 & 817 (93) & $3(0.34)$ & $59(7)$ & $1 / 153(1)$ & $2(0.3)$ \\
\hline Ear discharge & 15 & 5 & 21 & $21(100)$ & 0 & 0 & 0 & 0 \\
\hline BAL & 30 & 15 & 55 & $34(62)$ & $14(25)$ & $7(13)$ & $7 / 15$ (47) & $2(7)$ \\
\hline Throat & 16 & 8 & 30 & $15(50)$ & $12(40)$ & $3(10)$ & $2 / 8(25)$ & $7(44)$ \\
\hline Total & 773 & 179 & 985 & 887 (90) & $29(3)$ & $69(7)$ & $9 / 179(5)$ & $11(1)$ \\
\hline
\end{tabular}

a Fail to amplify in PCR-HRM

b Denominator no. specimens with multiple NTHi scrutinised

Table 3 Reclassification of isolates and specimens as $\mathrm{Hh}$ by the subgroups age and dwelling

\begin{tabular}{|c|c|c|c|c|c|c|}
\hline Specimen type & Subgroup & & $\begin{array}{l}\text { Hh isolate } \\
\text { reclassification, } n(\%)\end{array}$ & $p$ value & $\begin{array}{l}\text { Hh specimen } \\
\text { reclassification, } \mathrm{n}(\%)\end{array}$ & $p$ value \\
\hline $\mathrm{Np}$ & $\mathrm{Age}^{\mathrm{a}}$ & $\leq 1$ & $0 / 275(0)$ & & $0 / 218(0)$ & \\
\hline Isolates, $n=879$ & & $>1-3$ & $1 / 413(0.2)$ & 0.414 & 0/334 (0) & NA \\
\hline \multirow[t]{3}{*}{ Specimen, $n=712$} & & $>3-10$ & 2/191 (1) & 0.089 & $2 / 160(1)$ & 0.098 \\
\hline & Dwelling & Urban & $1 / 193(0.2)$ & 0.633 & $1 / 174(0.6)$ & 0.400 \\
\hline & & Remote & 2/686 (0.3) & & $1 / 538(0.2)$ & \\
\hline BAL & Age & $\leq 1$ & - & & & \\
\hline Isolates, $n=55$ & & $>1-3$ & $13 / 44(30)$ & 0.164 & $2 / 24(8)$ & 0.464 \\
\hline \multirow[t]{3}{*}{ Specimen, $n=30$} & & $>3-10$ & $1 / 11(9)$ & & $0 / 6(0)$ & \\
\hline & Dwelling & Urban & $5 / 10(50)$ & 0.049 & $0 / 4(0)$ & 0.556 \\
\hline & & Remote & $9 / 45(20)$ & & $2 / 26(8)$ & \\
\hline Throat & Age & $\leq 1$ & - & - & & \\
\hline Isolate, $n=30$ & & $>1-3$ & 8/23 (35) & 0.290 & 4/11 (36) & 0.377 \\
\hline \multirow[t]{3}{*}{ Specimen, $n=16$} & & $>3-10$ & $4 / 7(57)$ & & $3 / 5(60)$ & \\
\hline & Dwelling & Urban & $5 / 6(83)$ & 0.015 & $2 / 3(66)$ & 0.375 \\
\hline & & Remote & $7 / 24(29)$ & & $5 / 13(38)$ & \\
\hline
\end{tabular}

\footnotetext{
${ }^{a}$ Age $p$ values referenced to $\leq 1$
} 
otitis media undergoing grommet insertion surgery and 17 healthy controls, (266 isolates in total) 20.5 and $11.8 \%$ of Np presumptive NTHi isolates were reclassified by $16 \mathrm{~s}$ rRNA PCR as Hh, respectively [8]. However only $3.6 \%(4 / 110)$ of otitis prone and $0 \%$ of healthy control Np specimens were reclassified as having Hh-only. Differences in results could be real, or accounted for by multiple factors, such as geographic location, season, age, ethnicity, disease severity and density of Np colonisation, or speciation methodology. All studies included children $<36$ months. Neither ethnicity nor remoteness was reported in the WA or American cohorts. The Indigenous children in our studies (B, C and D) are at high risk of otitis media and have NTHi Np carriage rates of over $70 \%$ for most of their early childhood [2]. In this population the Hh may not be competitive, and therefore absent or not detected, in the Np.

In contrast to the $\mathrm{Np}$, significantly more BAL $(25 \%$, $14 / 55, \mathrm{p}<0.001)$ and throat $(40 \%, 12 / 30 ; \mathrm{p}<0.001)$ isolates were reclassified as $\mathrm{Hh}$. Throat specimens were less likely to be mixed (NTHi and Hh) and significantly more likely than BAL $(7 / 16,44 \%$ versus $2 / 30,7 \%)$ to be reclassified as Hh-only $(\mathrm{p}=0.003)$. These specimens were collected from children with a history of lung disease, and the higher Hh proportion in BAL specimens is consistent with that described in American adults with chronic obstructive pulmonary disease $(39.5 \%$ of sputum isolates were $\mathrm{Hh}$ ) [6]. For both BAL and throat specimens there was no significant difference in $\mathrm{Hh}$ prevalence by age group (BAL $\mathrm{p}=0.464$; throat $\mathrm{p}=0.377$ ), although there were no BAL nor throat samples from children aged $\leq 1$ year. In contrast to Np data, NTHi isolates from both the BAL and throat of urban children were significantly more likely be to be reclassified as Hh compared to remote children, $\mathrm{p}=0.049$ and 0.015 respectively (Table 3). This did not remain significant at the specimen level (BAL $p=0.556$; throat $\mathrm{p}=0.375$ ), due to high proportion of BAL and throat specimens with both NTHi and $\mathrm{Hh}$.

A small proportion $(69 / 985,7 \%)$ of isolates from $\mathrm{Np}$, BAL and throat specimens failed to amplify in the hpdbased PCR-HRM (Table 2). This was presumably due to considerable variation or absence of the $h p d$ gene as shown previously [13]. In one study up to $13 \%$ of $\mathrm{Np}$ and disease-related NTHi isolates were shown to be missing the hpd gene by whole genome sequencing [13]. This has potential implications for the efficacy of the pneumococcal $H$. influenzae protein $\mathrm{D}$ conjugate vaccine (PHiD-CV10) against H. influenzae, and for hpd-based diagnostics.

In conclusion, Hh prevalence varied markedly among respiratory specimen types. Our data support a recommendation for DNA-based discrimination of NTHi and
Hh for throat and BAL isolates, however, the low prevalence of $\mathrm{Hh}$ in nasal or nasopharyngeal swabs $(0.34 \%$ of presumptive NTHi) suggests that PCR discrimination is not routinely required for these specimens in this population. As the prevalence of $\mathrm{Hh}$ in other settings differs, population-specific recommendations for discrimination of Hh and NTHi are required to accurately determine the burden of disease attributable to NTHi. Multiple colony testing is recommended for throat and lung specimens as mixed NTHi and Hh populations can be expected.

\section{Limitations}

We have identified a wide variation in $\mathrm{Hh}$ prevalence between our studies and those reported. Due to low rates of positivity we do not recommend hpd based PCR methods for discrimination of NTHi and Hh in the Np in this population. Larger studies should be conducted to clarify best application of PCR methods for clinical specimens from the middle ear, lung and throat. It is unclear why Hh reclassification was concordant among lung specimens across studies, yet discordant among nasopharyngeal specimens. Differences in methodology (16S rRNA PCR $[6,8]$ in combination with MLST and DNA hybridisation [6], PCR-HRM [11]) may have contributed. Geographic location, remoteness of dwelling, variation in NTHi carriage and density and ethnicity could also contribute to this difference.

\section{Abbreviations}

NTHi: non-typeable Haemophilus influenzae; Hh: Haemophilus haemolyticus; Np: nasopharynx/nasal; ED: ear discharge; BAL: bronchoalveolar lavage; PCR: polymerase chain reaction; DNA: deoxyribonucleic acid; NT: Northern Territory; PCR-HRM: polymerase chain reaction high resolution melt; MLST: multi locus sequence testing.

\section{Authors' contributions}

$J B$ conceived the study, compiled and analysed the data, performed laboratory work and drafted the manuscript. $A B C$ and $A J L$ contributed to the acquisition of data. HSV, MJB, and RLM contributed to interpretation of the data. All authors contributed to the intellectual content of the manuscript. All authors read and approved the final manuscript.

\section{Author details \\ ${ }_{1}^{1}$ Menzies School of Health Research, Charles Darwin University, PO Box 41096, Casuarina, NT 0811, Australia. ${ }^{2}$ Department of Respiratory Medicine, Lady Cilento Children's Hospital, PO Box 3474, South Brisbane, QLD 4101, Australia. ${ }^{3}$ Institute of Health and Biomedical Innovation, Queensland University of Technology, GPO Box 2434, Brisbane, QLD 4001, Australia.}

\section{Acknowledgements}

We wish to thank the families who participated in these studies, and the Menzies Child Health Respiratory and Ear Health Research Program for collection of original specimens. Thanks to Koby Scarff for the laboratory support.

Competing interests

The authors declare that they have no competing interests. 


\section{Availability of data and materials}

The datasets analysed during the study are available from the corresponding author subject to necessary approvals.

\section{Consent for publication}

Not applicable.

\section{Ethics approval and consent to participate}

All studies included were approved by the Human Research Ethics Committee of Northern Territory Department of Health and Menzies School of Health Research and/or the Central Australian Human Research Ethics Committee when appropriate. Analyses performed in this study were under approvals 07-85 and 07-63. Samples from each child were collected after written informed consent was obtained from the child's parent or guardian.

\section{Funding}

The original studies were funded by NHMRC Grants (GNT436023, GNT545232, GNT1040830, and GNT605810) and GlaxoSmithKline. Neither the NHMRC nor GlaxoSmithKline had any input into study design or manuscript preparation. HSV is supported by a Fellowship under the NHMRC-funded HOT NORTH collaboration (GNT1131932). MJB and RLM are supported by NHMRC Early Career Fellowships (GNT1088733, GNT1034703). AJL is supported by a NHMRC Research Fellowship (GNT1020561). ABC is funded by a NHMRC Practitioner Fellowship (1058213).

\section{Publisher's Note}

Springer Nature remains neutral with regard to jurisdictional claims in published maps and institutional affiliations.

Received: 17 October 2017 Accepted: 10 May 2018

Published online: 21 May 2018

\section{References}

1. Van Eldere J, Slack MP, Ladhani S, Cripps AW. Non-typeable Haemophilus influenzae, an under-recognised pathogen. Lancet Infect Dis. 2014;14(12):1281-92. https://doi.org/10.1016/S1473-3099(14)70734-0.

2. Leach AJ, Wigger C, Beissbarth J, Woltring D, Andrews R, Chatfield MD et al. General health, otitis media, nasopharyngeal carriage and middle ear microbiology in Northern Territory Aboriginal children vaccinated during consecutive periods of 10-valent or 13-valent pneumococcal conjugate vaccines. Int J Pediatr Otorhinolaryngol. 2016;86:224-32. https //doi.org/10.1016/j.ijporl.2016.05.011.

3. Smith-Vaughan HC, Binks MJ, Marsh RL, Kaestli M, Ward L, Hare KM, et al. Dominance of Haemophilus influenzae in ear discharge from Indigenous
Australian children with acute otitis media with tympanic membrane perforation. BMC Ear Nose Throat Disord. 2013;13(1):12. https://doi. org/10.1186/1472-6815-13-12

4. Hare KM, Grimwood K, Leach AJ, Smith-Vaughan H, Torzillo PJ, Morris PS, et al. Respiratory bacterial pathogens in the nasopharynx and lower airways of Australian indigenous children with bronchiectasis. J Pediatr. 2010;157(6):1001-5. https://doi.org/10.1016/j.jpeds.2010.06.002.

5. Sethi S, Murphy TF. Bacterial infection in chronic obstructive pulmonary disease in 2000: a state-of-the-art review. Clin Microbiol Rev. 2001;14(2):336-63. https://doi.org/10.1128/CMR.14.2.336-363.2001.

6. Murphy TF, Brauer AL, Sethi S, Kilian M, Cai X, Lesse AJ. Haemophilus haemolyticus: a human respiratory tract commensal to be distinguished from Haemophilus influenzae. J Infect Dis. 2007;195(1):81-9. https://doi. org/10.1086/509824

7. Zhang B, Kunde D, Tristram S. Haemophilus haemolyticus is infrequently misidentified as Haemophilus influenzae in diagnostic specimens in Australia. Diagn Microbiol Infect Dis. 2014;80(4):272-3. https://doi. org/10.1016/j.diagmicrobio.2014.08.016.

8. Kirkham LA, Wiertsema SP, Mowe EN, Bowman JM, Riley TV, Richmond PC. Nasopharyngeal carriage of Haemophilus haemolyticus in otitis-prone and healthy children. J Clin Microbiol. 2010;48(7):2557-9. https://doi. org/10.1128/JCM.00069-10.

9. Leach AJ, Wigger C, Andrews R, Chatfield M, Smith-Vaughan H, Morris PS. Otitis media in children vaccinated during consecutive 7-valent or 10-valent pneumococcal conjugate vaccination schedules. BMC Pediatr. 2014;14:200. https://doi.org/10.1186/1471-2431-14-200.

10. Leach AJ, Mulholland EK, Santosham M, Torzillo PJ, Brown NJ, Mclntyre $P$, et al. Pneumococcal conjugate vaccines PREVenar13 and SynflorIX in sequence or alone in high-risk Indigenous infants (PREV-IX_COMBO): protocol of a randomised controlled trial. BMJ Open. 2015;5(1):e007247. https://doi.org/10.1136/bmjopen-2014-007247.

11. Pickering J, Binks MJ, Beissbarth J, Hare KM, Kirkham LA, Smith-Vaughan H. A PCR-high-resolution melt assay for rapid differentiation of nontypeable Haemophilus influenzae and Haemophilus haemolyticus. J Clin Microbiol. 2014;52(2):663-7. https://doi.org/10.1128/JCM.02191-13.

12. Price EP, Sarovich DS, Nosworthy E, Beissbarth J, Marsh RL, Pickering J, et al. Haemophilus influenzae: using comparative genomics to accurately identify a highly recombinogenic human pathogen. BMC Genomics. 2015;16:641. https://doi.org/10.1186/s12864-015-1857-x.

13. Smith-Vaughan HC, Chang AB, Sarovich DS, Marsh RL, Grimwood K, Leach $A J$, et al. Absence of an important vaccine and diagnostic target in carriage- and disease-related nontypeable Haemophilus influenzae. Clin Vaccine Immunol. 2014;21(2):250-2. https://doi.org/10.1128/CVI.00632 $-13$.

Ready to submit your research? Choose BMC and benefit from

- fast, convenient online submission

- thorough peer review by experienced researchers in your field

- rapid publication on acceptance

- support for research data, including large and complex data types

- gold Open Access which fosters wider collaboration and increased citations

- maximum visibility for your research: over 100M website views per year

At BMC, research is always in progress.

Learn more biomedcentral.com/submissions 